\title{
Hydrodynamic Data from Radar Observations
}

\author{
Caroline GAUTIER, the Netherlands \\ Deltares \\ Herman PETERS, the Netherlands \\ Rijkswaterstaat DID (Ministry of Infrastructure and the Environment)
}

Jos van HEESEN, the Netherlands

Nortek BV

Topic A) Innovations in acquisition techniques

\section{INTRODUCTION}

For a proper determination of hydraulic boundary conditions it is paramount that wave, current, water level and bathymetry measurements be available. On one hand, observations improve the understanding of flow and waves, the formulations and the models. On the other hand, observations are required in the statistical determination of extreme values. Besides, measurements are needed in the design of offshore and coastal structures and in operational forecasts used for instance in safe navigation, flood forecasting and ship routing.

Most commonly available measurements for such purposes are generally sparse, either in space (for instance point measurements of waves by buoys, or currents by ADCPs) or in time (for instance bathymetric survey campaigns).

However, ordinary marine radars, available at almost each port, have the potential to provide spatial wave, currents and bathymetric measurements in the regions they cover. Dedicated SeaDarQ software by Nortek processes the radar data and derives spatial information on waves, water depths and currents over a significant area. Their onshore position implies reliable operationality and limited maintenance costs. Existing radars and infrastructure are in general located in regions with complex geometries and complex wave, current and depth patterns (inlets, harbours); exactly the regions where data are most needed to validate and calibrate wave, flow and morphology models.

The lighthouse of Ameland - one of the Dutch islands in the Wadden Sea - is equipped with an Xband marine radar $(9.6 \mathrm{GHz})$. Since the beginning of 2010 this radar is connected to the dedicated SeaDarQ software. This paper describes the principle of X-band radar measurements and the application of the processed data.

\section{THE PRINCIPLE OF X-BAND RADAR FOR HYDRAULIC MEASUREMENTS}

$\mathrm{X}$-band radar refers to radar with electromagnetic energy ranging from approximately 8 to $12 \mathrm{GHz}$ in frequency. The wave length of the signal is a few centimetres. The method to derive hydrodynamical data from marine $X$-band radar is based on the reflection of the radar waves by small scale roughness features at the sea surface e.g. small capillary waves. In order to have enough capillary waves to produce a usable radar image, wind speeds are required in the range of about 2 to $20 \mathrm{~m} / \mathrm{s}$. This sea echo, known as sea clutter, is unwanted noise for navigational purpose. However, by virtue of an amplitude modulation mechanism, the sea clutter makes the wave crest patterns visible, with their associated wave lengths, velocities and orientations.

There are two reasons why the wave patterns are visible in the radar image. First of all, the roughness on a wave crest is higher than in a wave through due to hydrodynamic modulation. Second, the large gravity waves modulate the radar backscatter strength by tilting the sea surface and, for low grazing angles, the wave crests even create shadows at their back slopes. The latter 
mechanism only works for the range travelling waves (i.e. in the direction of the radar), while the former holds for both range travelling and azimuth travelling waves. This explains why the range travelling waves can be seen better in the radar image than the azimuth travelling waves.

The radar antenna on top of the Amelander lighthouse rotates continuously around a vertical axis and transmits intermittently short pulses of electromagnetic energy, the reflections of which are received up to a distance of a few kilometres. After about $0.1 \mathrm{~ms}$, when all the echoes of the previous pulse have died away, the next pulse is transmitted in a slightly different azimuth direction. It takes $2.85 \mathrm{~s}$ (and thus a few thousand pulses) to cover the full azimuth sector of $360^{\circ}$.

From a series (film) of radar images the wave propagation direction and the associated phase velocity can be estimated unambiguously. Hereto, the radar images need to be transformed from $(x, y, t)$ space via 3d FFT to $\left(k_{x}, k_{y}, \omega\right)$ space (with $k_{x}$, $k_{y}$ the orthogonal components of the wave number vector and $\omega$ the angular frequency). The ideal (simple) dispersion relation gives the relation between the wave length and the undisturbed phase velocity (no currents and deep water). However, in practice the phase velocity estimated from the sequence of radar images will often deviate from the undisturbed phase velocity. The measured difference makes it possible to compute the current velocity as well as the average water depth. To determine the water depth, mainly the low frequency part of the dispersion relation $(\mathrm{f}<0.15 \mathrm{~Hz}$ ) is used, while the high frequency part is mainly used to estimate the surface current vector. The measured current parameters represent the depth-averaged value of the vertical current profile in the upper few meters of the water column.

The radar spectra represent the radar intensity, and not the wave energy density. A Modulation Transfer Function (MTF) is required to assess the wave spectrum. This empirical function, linking the wave spectra with the radar spectra, depends on both the measuring system (video amplifier, antenna height, range compensation of the video signal) and environmental features (sea state, angle between waves and radar beam, distance to radar). In practice, it could be calibrated using buoy measurements.

(a)

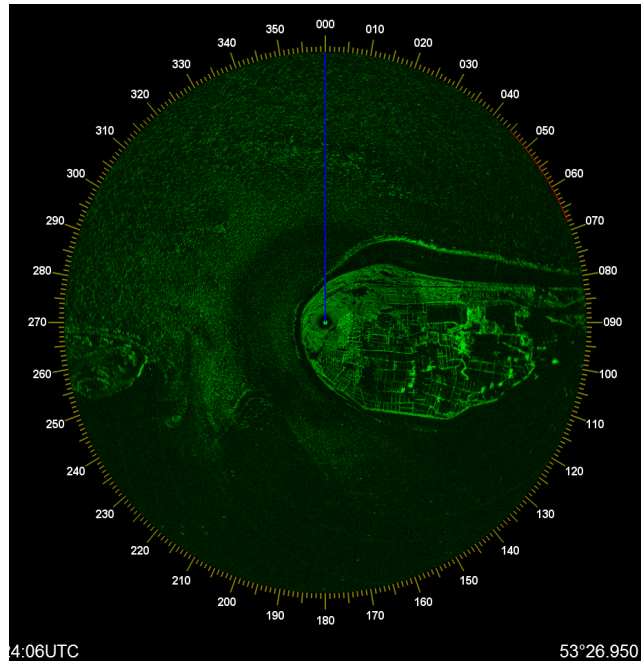

(b)

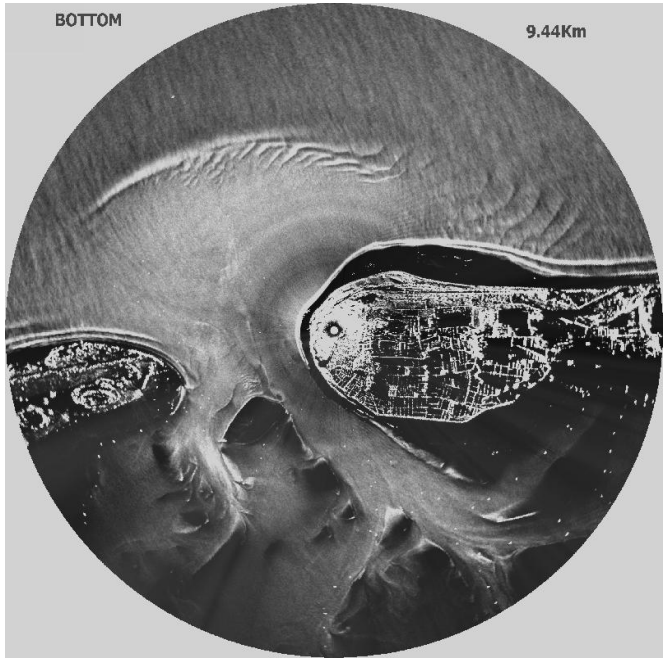

Figure 2.1: Unprocessed radar image (a) and processed snapshot (b), both at Ameland

Depending on the actual settings, the radar image may consist of rectangular pixels with ca. $7.5 \mathrm{~m}$ resolution, which form a calculation matrix of the underlying radar resolution cells. The radar range resolution $\Delta R$ depends on the pulse length $\Delta T\left(\Delta R=0.5^{*} c^{*} \Delta T\right.$, with $\mathrm{c}=3^{*} 10^{8} \mathrm{~m} / \mathrm{s}$; speed of light). The azimuthal resolution $\Delta L$ depends on the radar beam width and thus on the horizontal length $D$ of the antenna $\left(\Delta L=R^{*} \lambda / D\right)$ with $R$ the distance from the radar and $\lambda$ the wave length of the radar signal, being $0.03 \mathrm{~m}$. The radius of the radar image consists of 1000 pixels $(7.5 \mathrm{~km})$. 
In case of the Amelander radar, the radar intensity spectra as well as wave and current parameters are computed for rectangular areas with size $959 \mathrm{~m} \times 959 \mathrm{~m}$. The spatial resolution for the estimated spectra and the parameters derived thereof is ca $300 \mathrm{~m}$. Whereas spectra from buoys are based on time series (for instance 2048 values with $0.5 \mathrm{~s}$ interval) at one location, the radar spectra are based on a shorter period and more locations (for instance $128 \times 128$ pixels $\times 32$ images, equivalent to about $90 \mathrm{~s}$ of radar measurements). Since the FFT requires rectangular pixels, the original polar pixels are linearly interpolated to rectangles.

A 'snapshot' represents the moving average of a number (for instance $8,16,32,64$ ) of successive radar images. In this average view the waves are no longer visible, but bathymetric characteristics as channel positions and other fixed items as e.g. marking buoys emerge more clearly, see Fig. 2.1.

The following parameters can be derived from the radar measurements: Wave frequency, wave length, wave speed, wave direction, wave height (after calibration using a wave buoy), current velocity and water depth.

\section{APPLICATION OF RADAR DATA IN A WAVE HINDCAST}

\section{$\underline{3.1 \text { Introduction }}$}

The radar data of Ameland is part of the SBW measuring campaign by the Dutch Ministry of Infrastructure and the Environment. SBW stands for the "Strength and Loading of Water Defenses" Program, filling in the knowledge gaps in the periodic safety assessment of the primary flood defenses. One of the research topics within SBW is the penetration of North Sea waves into the tidal inlet systems of the Wadden Sea. Aim is to ultimately improve the numerical wave model SWAN (Booij et al., 1999) on this point. The radar data is not used as ground truth yet, but by using the data, experience is gained concerning its reliability and usability, see also Deltares, 2011.

\subsection{Data}

The hindcast presented here concerns the northwestern storms of October 20 and 24, 2010, starting with a strong breeze (6-7 Bft). The significant wave height at the most northern buoys AZB11 and AZB12 was over $4 \mathrm{~m}$. We selected a strong ebb situation (9:10 (T2)). The next storm occurred a few days later on October 24th. This storm was more severe, both in terms of wind, waves and water levels. The wind ("gale", 8 Bft, wind speed above $20 \mathrm{~m} / \mathrm{s}$ ) turned from west to north-northwest. The offshore buoys AZB11 and AZB12 observed significant wave heights of over $5 \mathrm{~m}$. We selected the maximum off shore wave height in combination with flood currents (6:00 (T6)).

Besides the radar data (covering about half a circle with $15 \mathrm{~km}$ diameter and a time window of about 13 minutes), twelve waverider buoys are present in the tidal inlet of Ameland, of which a subset of three within the range of the radar (see Figure 3.1). Offshore wave data is available at the wave buoys SON and ELD. Water levels have been measured at Terschelling Noordzee (TNZ) and Nes. Wind data is available from Huibertgat (Huib), Terschelling Noordzee en Wierumergronden (Wier). Use is made of bathymetry data measured in the period March - September 2010, completed with bathymetry data from before 2008.

\subsection{SWAN wave model}

Stationary SWAN simulations were carried out for several events, of which we only present the selected times T2 and T6. On a larger grid (G1) the eastern and western boundary conditions for the nested detail grid (G2) of the Amelander inlet are computed. Besides these, the nested grid receives observed wave conditions on its northern boundary. For simulations on the scale of the Amelander inlet, stationary simulations are appropriate. 


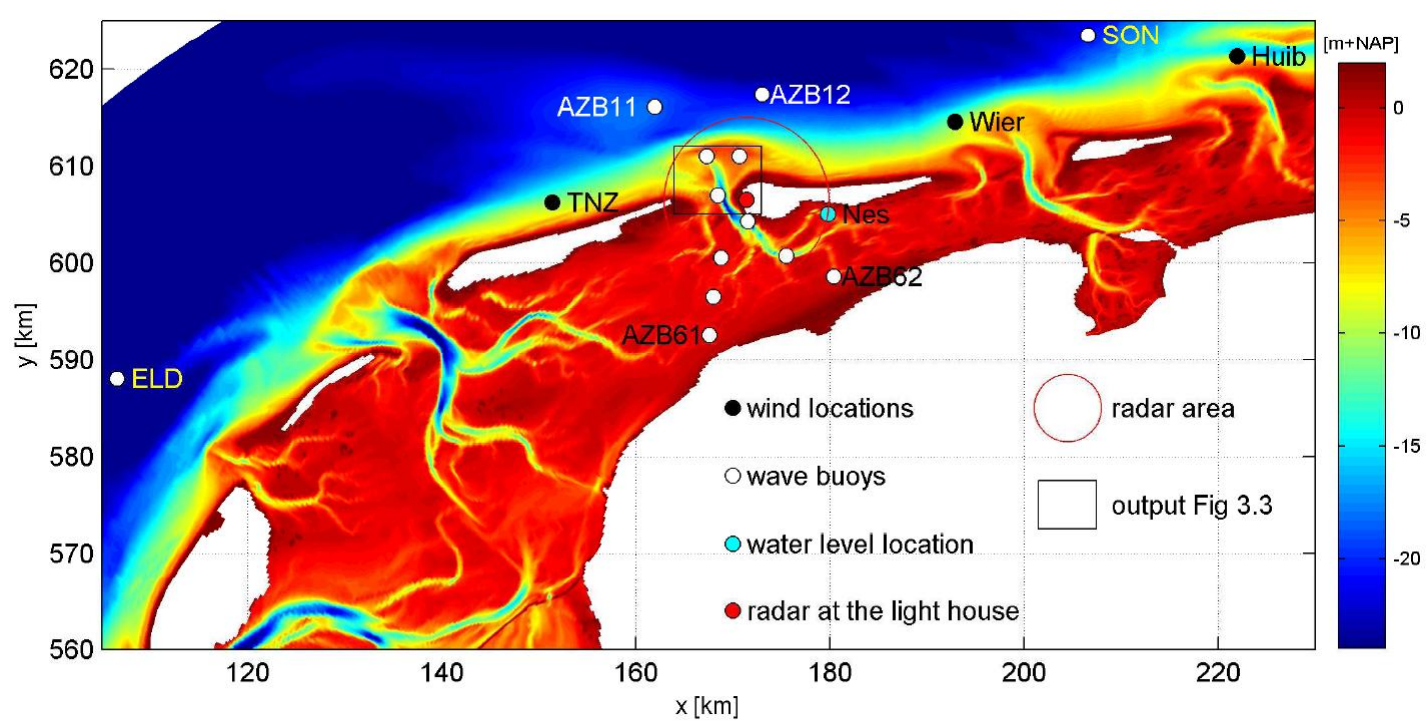

Figure 3.1: Measurement locations and bathymetry Amelander tidal inlet

The model input consists of bathymetric data, curvi linear computational grids, wave boundary conditions based on observations at ELD, SON, AZB11 and AZB12, uniform wind based on observations at Wierumergronden, uniform water levels observed at Nes (used on G1 only) and nonuniform water level and current fields computed by Delft3D (Deltares, 2012) used on G2. The computations were performed with SWAN version 40.81 in third generation mode. The physical settings are equal to the so called WTI-2011 settings, which were used to derive the Hydraulic Boundary Conditions 2011.

\section{$\underline{3.4 \text { Results }}$}

First, the SWAN results for the spectral significant wave height $\mathrm{H}_{\mathrm{m} 0}$ are compared with the buoy measurements, to show that the agreement - in spite of a limited overestimation - is good, see Figure 3.2.
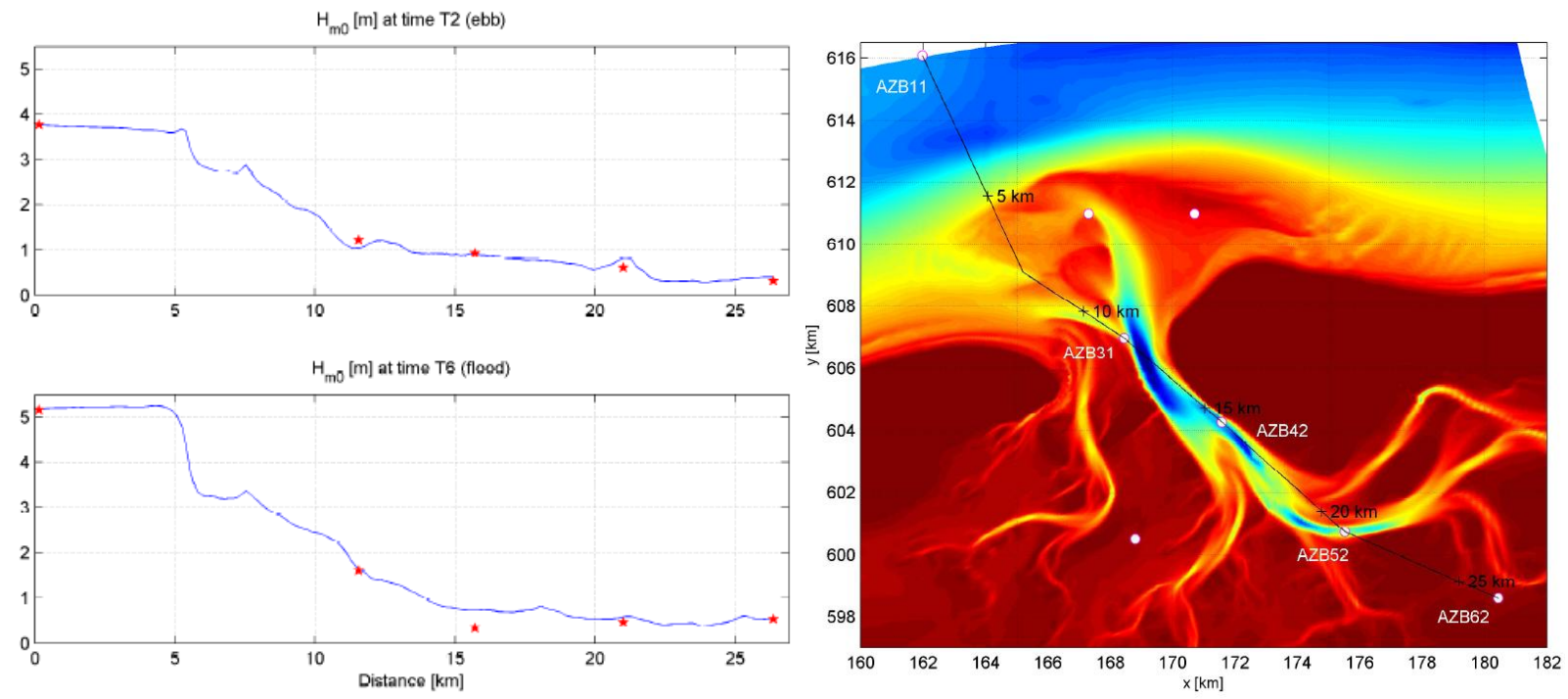

Figure 3.2: (left) SWAN model results wave height $H_{m 0}$ for times T2, T6. red pentagons indicate measurements at buoys AZB11, AZB31, AZB42, AZB52, AZB62. (right) location transect. 
Next, the radar data are compared to the SWAN results, to obtain an indication of their agreement. Note however that both the radar observations and the SWAN results have their uncertainties. Where differences between the two sources occur, it is unknown which of them is closer to reality. Where possible, the buoy data will also be included.

The radar data contain valuable information but should be used with care. In particular for areas with large bottom gradients, the results are less reliable. This is primarily due to the spatial averaging over the relatively large resolution. The dominant wave direction is considered, this is the direction of the energy bin with maximum energy as function of direction and frequency. For SWAN, the dominant direction has been computed accordingly, from the absolute $2 \mathrm{~d}$-wave spectra.
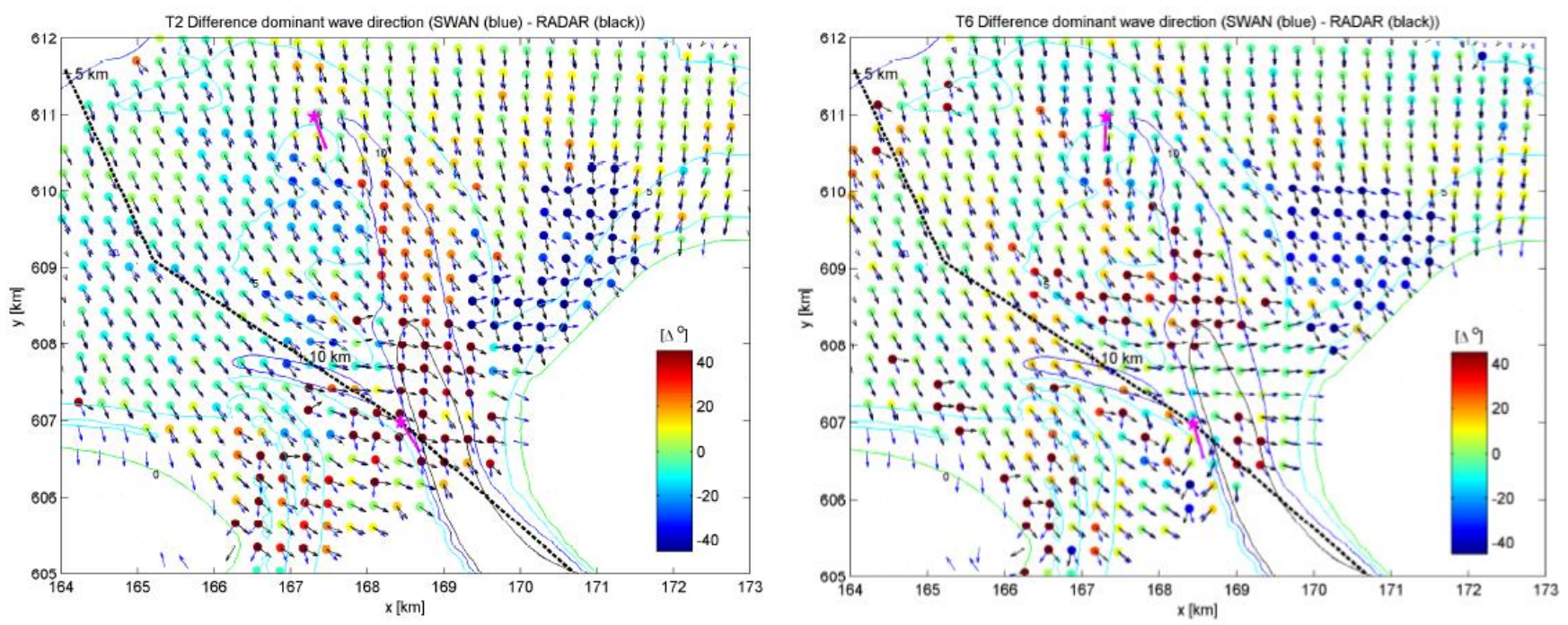

Figure 3.3: Difference dominant wave direction SWAN-RADAR for T2 (ebb) and T6 (flood)
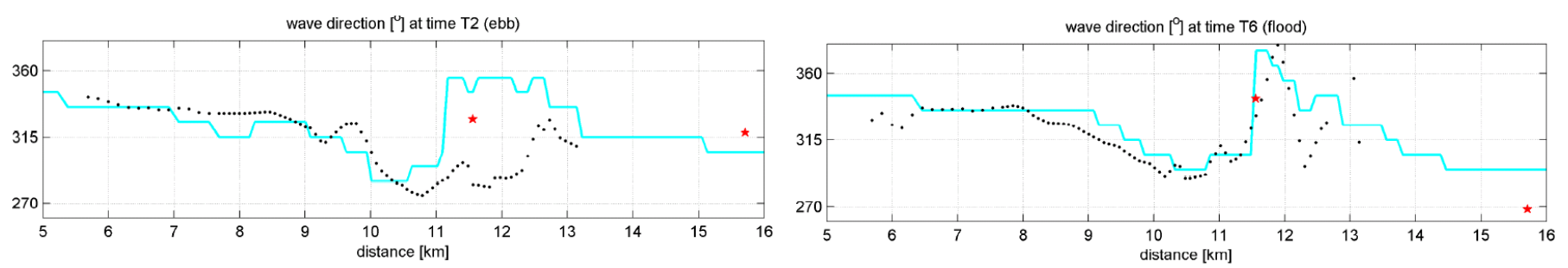

Figure 3.4: SWAN (blue line) and radar (black dots) dominant wave direction on transect (see Figure 3.3 for its position) for times T2, T6. Red pentagons are measured peak wave directions at buoys AZB31 and AZB42.

In Figure 3.3 the wave direction from the radar observations and from SWAN on identical locations are compared for the selected times. The blue arrows represent the wave direction as computed by SWAN, the black arrows refer to the radar data. The colors indicate the difference between the two sources (SWAN-radar). The majority of the area, especially outside of the inlet, turns out to be fairly green, which means that the directional differences between the radar and SWAN are small. However, moving into the inlet and to the north west shore of Ameland, some large differences are found. According to the SWAN simulations, the waves do not cross the tidal channel $(X=169 \mathrm{~km} ; Y=607.5$ $\mathrm{km}$ ) in all situations. According to the dominant wave direction of the radar the waves do cross in all considered cases. Since both sources have their uncertainties, it is unknown whether SWAN or the radar performs better here.

In Figure 3.4 not only SWAN and radar data are present, but also peak directions according to waveriders AZB31 and AZB42 are included. On large stretches - from $\mathrm{km} 5$ to $\mathrm{km} 10$ - the agreement 
between SWAN and radar wave direction is good. In both cases, the wave direction changes over this stretch roughly from $340^{\circ} \mathrm{N}$ to $300^{\circ} \mathrm{N}$. The part of the transect past $\mathrm{km} 10$ shows a rather variable directional situation. This is due to larger spatial variation in bathymetry (and hence wave direction) and also due to the bimodality of the wave spectra here. The results at time T6 (flood) look good, with agreement between SWAN and radar and even with buoy AZB31. Just past the buoy at km 12 the wave direction has its maximum $\left(360-380^{\circ} \mathrm{N}\right)$, as the waves refract out of the steep channel here. However, this does not occur on all times. Even with the buoy measurements included it is hard to judge the radar and SWAN results on the transect for such a locally varying parameter. The differences with the buoy observations are quite large, as is the spatial variation of SWAN and the radar.

\section{DISCUSSION}

The radar observations provide valuable measurements for waves, currents and bathymetry. The main advantage over point measurements is the spatial coverage. This paper focused on the wave analysis at Ameland. However, in the Netherlands there are also SeaDarQ systems available at Kijkduin and Hook of Holland. The latter has the task to monitor the currents for vessels entering the Port of Rotterdam. Since radar provides bathymetric data even during storms, when survey vessels can not sail out, both long term and short term morphologic changes can be derived.

It is important to know the definitions of the parameters in order to prevent inconsistent comparisons. The radar data is based on dominant wave parameters, referring to the maximum energy bin as function of direction and frequency. Dominant direction may shift abruptly if the variance in each directional lobe is about equal. For bimodal spectra it is difficult to catch the wave direction in one value. Small spectral differences could lead to large difference in the dominant parameters. Since the SWAN spectra show bimodality (not shown here), it is likely that this is the main reason for the rather large differences between SWAN and the radar here (see Fig 3.3).

\section{CONCLUSIONS}

Overall, the agreement between the wave direction of radar and SWAN is good. In areas with large spatial variation in wave direction the radar data seems to be less reliable. Some times, large differences exist between SWAN and the radar observations in the main channel. According to the radar, the waves always cross the channel, whereas in SWAN the dominant waves follow in these cases the direction of the channel, instead of crossing it. Since both sources have their uncertainties, it is unknown whether SWAN or the radar performs better here. Probably, the spectra are bimodal here, and the dominant wave direction can shift abruptly if the variance in each directional lobe is about equal.

The radar observations provide valuable measurements. However, this innovative technique is not yet up to such standards that it can replace other wave and current measurements. The data analysis shows that more experience must be gained in order to benefit from all its possibilities. Based on the first experiences with the radar data we hope that in the future the radar data will allow us to calibrate our hydrodynamic models for both water levels and currents, and provide proper insight in the wave models being applied in complex areas like the tidal inlet of Ameland.

\section{REFERENCES}

Booij, N., R.C. Ris and L.H. Holthuijsen. 1999. "A third generation wave model for coastal regions, Part I, Model desciption and valdiation". J. Geophys. Res., 104, C4, 7649-7666.

Deltares. 2011. "Comparison SWAN, PHAROS and radar wave observations." Deltares report 1204199002-HYE-0009 dd 24 November 2011.

Deltares. 2012. "Water level modelling of storm events in the Wadden Sea." Deltares report 1204199003-HYE-0007 dd 10 January 2012. 